\title{
Involvement of Sp1 in Butyric Acid- Induced HIV-1 Gene Expression
}

\author{
Kenichi Imaia Takashi Okamoto $^{\mathrm{b}}$ Kuniyasu Ochiai ${ }^{\mathrm{a}}$ \\ aDepartment of Microbiology, Division of Immunology and Pathobiology, Dental Research Center, \\ Nihon University School of Dentistry, Tokyo, 'Department of Molecular and Cellular Biology, Nagoya \\ City University, Graduate School of Medical Sciences, Nagoya, Japan
}

\section{Key Words}

HIV-1 $\bullet$ Butyric acid $\bullet$ Sp $1 \cdot$ HDAC

\begin{abstract}
Background/Aims: The ability of human immunodeficiency virus-1(HIV-1) to establish latent infection and its re-activation is considered critical for progression of HIV-1 infection. We previously reported that a bacterial metabolite butyric acid, acting as a potent inhibitor of histone deacetylases (HDACs), could lead to induction of HIV-1 transcription; however, the molecular mechanism remains unclear. The aim of this study was to investigate the effect of butyric acid on HIV-1 gene expression. Methods: Butyric acid-mediated HIV-1 gene expression was determined by luciferase assay and Chromatin immunoprecipitation assay. Western blot analysis and ELISA were used for the detection of HIV-1. Results: We found that Sp1 binding sites within the HIV-1 promoter are primarily involved in butyric acid-mediated HIV-1 activation. In fact, Sp1 knockdown by small interfering RNA and the Sp1 inhibitor mithramycin A abolished the effect of butyric acid. We also observed that CAMP response element-bindingbinding protein (CBP) was required for butyric acid-induced HIV-1 activation. Conclusions: These results suggest that butyric acid stimulates HIV-1 promoter through inhibition of the Sp1-associated HDAC activity and recruitment of CBP to the HIV-1 LTR. Our findings suggest that Sp1 should be considered as one of therapeutic targets in anti-viral therapy against HIV-1 infection aggravated by butyric acid-producing bacteria.
\end{abstract}

Copyright (C) 2015 S. Karger AG, Basel

\section{Introduction}

Human immunodeficiency virus-1 (HIV-1) is the causative agent of acquired immunodeficiency syndrome (AIDS). It is well established that the extents of its in vivo replication and the viral load are well correlated with the rate of disease progression. Current anti-retroviral therapy (ART) protocols consist of combinations of various antiretroviral agents that can efficiently decrease the viral load to below the detection limit, thus 
reducing the AIDS mortality rate [1-3]. However, despite the potency of ARTs, latent HIV-1 infection can escape from host immune responses and anti-retroviral therapy. These latently infected cells are permanent sources of viral re-activation that can lead to rebound of the viral load after interruption of ART [1,4]. Therefore, the viral latency is the main obstacle in eradication of HIV-1 from patients. Thus, elucidation of the molecular mechanisms involved in the maintenance and cessation of HIV latency are required to comprehensively illustrate the pathophysiology of HIV-1 infection in order to develop preventive measures and novel therapies.

HIV-1 gene expression is the major determinant of viral replication leading to progression of AIDS. After HIV-1 infection, integrated HIV-1 proviral DNA is incorporated into the host nucleosomes, where the transcriptional activity of viral long terminal repeat (LTR) is under the control of local nucleosomal structure [5-8]. Evidences have indicated that epigenetic modifications of the LTR play regulatory roles in the induction of HIV-1 transcription and viral expression [2, 5, 8, 9]. Previous studies have shown that the presence of histone deacetylases (HDACs) leads to the repression of HIV-1 LTR, whereas inhibition of HDACs by HDAC inhibitor (HDACi) results in up regulation of HIV-1 gene expression $[2,5,8]$. HDAC1 mediates chromatin remodelling resulting in repression of both HIV-1 transcriptional activity and viral replication [8, 10-13]. Negative transcription factors such as ying-yang protein 1-late SV40 factor (YY1-LSF) complex, nuclear factor- $\kappa B(\mathrm{NF}-\kappa \mathrm{B}) \mathrm{p} 50$ homodimer, C-promoter-binding factor and the specificity protein 1 (Sp1)-c-Myc complex have been shown to mediate recruitment of HDAC1 to the LTR and, consequently, inhibit transcription from the HIV-1 promoter [10-13]. We also reported that activator protein 4 (AP-4) acts as a transcriptional repressor by recruiting HDAC molecules and is involved in the maintenance of viral latency [14].

On the other hand, many reports have shown that butyric acid, an anaerobic metabolite of certain bacteria [15], is a potent inhibitor of HDAC, which leads to alterations in chromatin structure and diminished HIV-1 gene expression [16-18]. Kashanchi et al. [19] demonstrated augmentation of HIV-1 replication by butyric acid in latently infected primary mononuclear cells from HIV-1 infected individuals as well as HIV-1 latently infected cell lines, such as OM10.1, ACH-2 and U1. We previously demonstrated that butyric acid-producing bacteria in gut, oral cavities and vagina can strongly induce histone acetylation and HIV-1 replication from latently infected cells by inhibiting $\operatorname{HDAC}[20,21]$; thus, co-infection with anaerobic bacteria is an important risk factor for progression of AIDS among people infected with HIV1. However, the molecular mechanism of butyric acid activation of HIV-1 gene expression has not been elucidated.

In the present study, we investigated the effect of butyric acid on HIV-1 gene expression and demonstrated that Sp1 plays a key role in butyric acid-induced HIV-1 gene expression.

\section{Materials and Methods}

Reagents

Butyric acid was purchased from Wako Pure Chemical Industries, Ltd. (Osaka, Japan). Human recombinant tumour necrosis factor- $\alpha$ (TNF- $\alpha$ ) was purchased from Roche Diagnostics Deutschland GmbH (Mannheim, Germany) and used at a concentration of $3.0 \mathrm{ng} / \mathrm{mL}$ for NF- $\kappa \mathrm{B}$ stimulation. Mithramycin A and C646 were purchased from Sigma (St. Louis, MO, USA). Antibodies for Sp1, CBP and the acetylated form of human histone H3 (Ac-H3) were obtained from Upstate Biotechnology, Inc. (Lake Placid, NY, USA), anti-HDAC1, c-myc, RNA polymerase II (RNAPII) and normal rabbit anti-IgG were obtained from Santa Cruz Biotechnology, Inc. (Santa Cruz, CA, USA), and anti-AP-4 antibody was obtained as previously described [14].

Cell culture and HIV-1 latently infected human cell lines

ACH-2 is a chronically HIV-1-infected T-cell line derived from the parental cell line A3.01 [22, 23] that was obtained from the AIDS Research and Reference Reagent Program (National Institute of Allergy and Infectious Diseases, National Institutes of Health, Bethesda, MD, USA) and maintained at $37^{\circ} \mathrm{C}$ in Roswell 


\section{Cellular Physiology Cell Physiol Biochem 2015;37:853-865 \\ \begin{tabular}{l|l|l} 
DOI: 10.1159/000430213 & (C) 2015 S. Karger AG, Basel
\end{tabular} \\ \begin{tabular}{l|l|} 
and Biochemistry Published online: September 18, 2015 & www.karger.com/cpb \\
\hline
\end{tabular}}

Imai et al.: Sp1 Plays a Key Role in Butyric Acid-Mediated HIV-1 Activation

Park Memorial Institute 1640 medium (Sigma) with 10\% heat-inactivated foetal bovine serum (Sigma), penicillin $(100 \mathrm{U} / \mathrm{mL})$ and streptomycin $(100 \mu \mathrm{g} / \mathrm{mL})$. To maintain HIV-1 latency in ACH-2 cells, $20 \mu \mathrm{M}$ azidothymidine (Sigma) was added to the culture medium but was excluded prior to conducting the experiments. The cells $\left(2.0 \times 10^{6}\right.$ cells $/ 2.0 \mathrm{~mL}$ medium/well $)$ were treated with butyric acid. HeLa cells and TZM-bl cells, a HeLa-derived cell line expressing surface CD4, CXCR4 and CCR5 containing a chromatinintegrated HIV-1 LTR $[24,25]$ were grown at $37^{\circ} \mathrm{C}$ in Dulbecco's modified Eagle's medium (Sigma) with $10 \%$ heat-inactivated foetal bovine serum.

\section{Construction of plasmids}

Construction of HIV-1 LTR-based luciferase expression plasmid: wild-type (WT CD12-luc) HIV-1 LTRLuc (containing the HIV-1 LTR U3 and R regions), its 5' truncated mutant CD23-Luc (containing positions -117 to +80 of HIV-1 LTR), CD52-Luc (containing positions -65 to +80 of HIV-1 LTR) and the NF-KB mutant HIV-1 LTR-Luc, where NF- $\mathrm{KB}$ sites were mutated in WT HIV-1 LTR-Luc, were prepared as previously described [26-28]. Sp1, AP-4 or LSF mutant HIV-1 LTR-Luc plasmids were generated by PCR using a QuikChange site-directed mutagenesis kit (Stratagene, Inc., La Jolla, CA, USA) as described previously [14, 26]. The following mutant sequences were utilized: Sp1 mutant HIV-1 LTR-Luc, TCG ttA; AP4 mutant HIV1 LTR-Luc, CAG CTC; LSF mutant HIV-1 LTR-Luc, CAG tcG (consensus sequences of the binding sites are underlined and the mutated sequences are denoted by lower case letters). All constructs were confirmed by dideoxynucleotide sequencing using the ABI PRISM ${ }^{\circledR}$ dGTP BigDye ${ }^{\mathrm{TM}}$ Terminator v3.0 Ready Reaction Cycle Sequencing kit (PerkinElmer, Inc., Waltham, MA, USA) on an ABI PRISM ${ }^{\circledR} 373$ DNA Sequencer (Applied Biosystems, Foster City, CA, USA). Sp1-Luc [29] was kindly provided by Dr. K. Kohno.

\section{Transfection and luciferase assay}

HeLa cells $\left(1 \times 10^{5}\right.$ cells $\left./ \mathrm{mL}\right)$ were cultured in 12-well plates and HIV-1 LTR-based reporter plasmids (20 ng) described above, together with $10 \mathrm{ng}$ of the internal control plasmid pRL-TK (Promega, Fitchburg, WI, USA) expressing Renilla luciferase, were transfected with FuGENE 6 transfection reagent (Roche) according to the manufacturer's protocol $[14,26]$. After $24 \mathrm{~h}$ of transfection, the cells were treated with butyric acid (1.5 mM) or TNF- $\alpha$ ( $3 \mathrm{ng} / \mathrm{mL}$ ), as a positive control, for an additional $24 \mathrm{~h}$. These cells were then harvested using Passive Lysis Buffer (Promega), and the extracts were assessed for luciferase activity using the Dual-Luciferase Assay System (Promega). The luciferase activity was normalized by Renilla luciferase activity for transfection efficiency. All experiments were performed in triplicate and the data are presented as the fold increase in luciferase activity (mean \pm SD) relative to controls.

\section{Western blot analysis}

The Western blot protocol was described previously [26, 28]. Briefly, the cells were washed once with ice-cold phosphate-buffered saline and harvested with lysis buffer (Cell Signaling Technology, Inc., Danvers, MA, USA). Cellular debris was removed by centrifugation at $15000 \mathrm{~g}$ for $10 \mathrm{~min}$. After centrifugation, the supernatant proteins were separated by SDS-PAGE and transferred to a polyvinylidene fluoride membrane (EMD Millipore Corporation, Billerica, MA, USA). The Pierce Microplate BCA Protein Assay kit-Reducing Agent Compatible kit (Thermo Scientific, Inc., Rockford, IL, USA) was used to standardise the protein concentration in all samples. The membrane was probed with respective antibodies and immunoreactive proteins were visualised using the SuperSignal West Pico enhanced chemiluminescence kit (Thermo Scientific, Inc.). To detect HIV-1 proteins, the cell lysates were subjected to immunoblotting using sera collected from AIDS patients [20, 26].

\section{RNA interference}

Short interfering (si) RNAs against Sp1 and control GFP were purchased from Santa Cruz Biotechnology, Inc. and Takara Bio., Inc. (Otsu, Japan), respectively. TZM-bl cells were cultured in 12-well plates and treated with $100 \mathrm{nM}$ siRNAs using Lipofectamine RNAiMAX reagent (Invitrogen Corporation, Carlsbad, CA, USA) according to the manufacturer's instructions and incubated for additional $48 \mathrm{~h}$. The cells were then incubated for another $8 \mathrm{~h}$ in the presence or absence of butyric acid $(1.5 \mathrm{mM})$. Western blotting was performed with anti-Sp1 antibody to confirm knockdown of the Sp1 protein. The transfected cells were harvested and assessed for luciferase activity.

\section{KARGER}




\section{Cellular Physiology Cell Physiol Biochem 2015;37:853-865 \\ \begin{tabular}{l|l|l}
\hline DOI: 10.1159/000430213 & (C) 2015 S. Karger AG, Basel
\end{tabular} \\ \begin{tabular}{l|l|} 
and Biochemistry Published online: September 18, 2015 & www.karger.com/cpb \\
\cline { 1 - 2 }
\end{tabular}}

Imai et al.: Sp1 Plays a Key Role in Butyric Acid-Mediated HIV-1 Activation

Chromatin immunoprecipitation (ChIP) assay

ChIP assays were performed as described previously using the Magna ChIP assay kit (EMD Millipore Corporation) $[20,26]$. Briefly, TZM-bl cells $\left(2 \times 10^{6}\right)$ were fixed with $1 \%$ formaldehyde at $37^{\circ} \mathrm{C}$ for $10 \mathrm{~min}$ and the reaction was stopped by the addition of $125 \mathrm{mM}$ glycine. The cells were lysed and the cross-linked chromatin was sheared by sonication 10 times for $30 \mathrm{~s}$ each at maximum power with $30 \mathrm{~s}$ of cooling on ice between pulses (Bioruptor; Cosmo Bio Co., Ltd., Tokyo, Japan). Sheared chromatin fractions were collected and pre-cleared with salmon sperm DNA and protein G-agarose beads for $1 \mathrm{~h}$ followed by immunoprecipitation with the desired antibodies at $4^{\circ} \mathrm{C}$ overnight with rotation. The precipitated DNA was analysed by PCR (35 cycles) with HotStarTaq Master Mix (Qiagen, Hilden, Germany) and primers for HIV-1 LTR (-176 to +61; forward, 5'-CGA GAC CTG CAT CCG GAG TA-3'; reverse, 5' -AGT TTT ATT GAG GCT TAA GC- $3^{\prime}$ ). PCR products were separated by $2 \%$ SDS-PAGE. For each reaction, $10 \%$ of the original sheared chromatin DNA was similarly reversed cross-linked and purified, and the recovered DNA was used as the input control.

\section{Measurement of viral p24 antigen}

The stimulatory effect of butyric acid in ACH-2 cells was evaluated based on the extent of HIV-1 p24 core antigen production as previously described $[20,21,28]$. Briefly, cells $\left(2 \times 10^{6}\right)$ were incubated with or without butyric acid for $24 \mathrm{~h}$ at $37^{\circ} \mathrm{C}$. The culture supernatants were then collected and measured for viral p24 antigen concentrations using the Retrotek HIV-1 p24 antigen enzyme-linked immunosorbent assay (ELISA) kit (Cellular Products, Buffalo, NY, USA). All experiments were performed in triplicate and the data are presented as means \pm SD.

\section{Results}

\section{Effects of butyric acid on HIV-1 LTR transcription}

As we previously reported, butyric acid could activate HIV-1 replication from latently infected cells in a dose-dependent manner $[20,21]$. To determine the mechanism of how butyric acid activates HIV-1 gene expression, we generated various plasmid constructs, as shown in Fig. 1A, and transfected each construct into HeLa cells to determine the level of HIV-1 gene expression using luciferase assay. The wild-type (WT) HIV-1 LTR promoter (CD12-luc) contains binding sites for several transcription factors, including AP-1, Myb, nuclear factor of activated T-cells-1 (NFAT1), cytoplasmic 2, NF- $\kappa B$, Sp1, TATA-box binding protein, AP-4 and LSF (Fig. 1A). As shown in Fig. 1B, butyric acid (1.5 mM) stimulated the WT HIV-1 LTR promoter in a dose-dependent manner. TNF- $\alpha(3 \mathrm{ng} / \mathrm{ml})$ could also activate HIV-1 gene expression (positive control) but to a much lesser extent. Butyric acid could stimulate the CD23-Luc HIV-1 LTR promoter, although devoid of the upper sequence elements from -117, similarly to WT HIV-1 LTR (Fig. 1C). Therefore, the AP-1, Myb and NF-AT1 binding sites of HIV-1 LTR were not essential for butyric acid-induced HIV-1 gene expression. The activation of NF- $\mathrm{KB}$ binding to the HIV-1 LTR is the most critical step for cytokine-stimulated

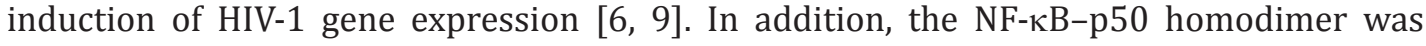
shown to mediate HDAC1 recruitment to the LTR and, consequently, inhibited transcription of the viral promoter [12]. Thus, we first examined whether NF- $\mathrm{BB}$ is involved in butyric acid-induced HIV-1 transcription. When NF- $\mathrm{KB}$ binding sites in LTR were eliminated (CD52Luc), marked transcriptional activation by butyric acid was still observed, whereas it totally abolished the effects of TNF- $\alpha$ (Fig. 1D). To confirm the role of the NF- $\kappa B$ binding sites in butyric acid-induced HIV-1 gene expression, the HIV-1 LTR construct lacking two NF- $\kappa B$ binding sites (NF- $\mathrm{kB}$ mut HIV-1 LTR-Luc) was transfected into HeLa cells. When these cells were stimulated with butyric acid, significant induction by butyric acid was still observed (Fig. 1E). These results clearly indicate that transcriptional control elements in adjacent to the HIV-1 mRNA start site within the LTR, but not the NF- $\mathrm{kB}$ binding sites, are responsible for the butyric acid-induced HIV-1 gene expression. 
Fig. 1. The effect of butyric acid on HIV-1 LTR transcription. (A) A schematic diagram of the HIV-1 LTRbased reporter constructs used to determine the contribution of various portions of the HIV-1 promoter. CD12-Luc contains the full sequences of the $\mathrm{U} 3$ and $\mathrm{R}$ regions. CD23-Luc and CD52-Luc encompass the downstream sequence from position -117 (the HIV-1 LTR cap site) and that from position -65 , respectively. NF$\kappa \mathrm{B}$ mutant HIV-1 LTR-Luc was mutated at the NF- $\mathrm{KB}$ binding sites of the WT HIV-1 LTR-Luc. (B-E) Results of luciferase assays using HIV-1 LTRs of various mutants. The HIV-1 LTR luc reporter constructs described in (A) expressing the luciferase gene under the control of HIV-1 LTR at 20 ng were transfected into HeLa cells, which were incubated for $24 \mathrm{~h}$ and stimulated with either butyric acid (BA; $1.5 \mathrm{mM}$ ) or TNF- $\alpha$ (3 ng/ml) for additional $24 \mathrm{~h}$. The luciferase activity of each cell lysate was then measured. All experiments were performed in triplicate and the data are presented as the fold increase in luciferase activity (means \pm S.D.) relative to the control.

The Sp1 binding site is required for butyric acid-induced HIV-1 LTR gene expression

Butyric acid inhibits the enzymatic activity of HDAC by competing with the HDAC substrate at the enzyme catalytic centre [18], stimulating transcription of various genes including HIV-1 $[15,20,21]$. Three tandem repeats of putative Sp1-binding sites (GC) (position -69 to -78 , position -58 to -67 and position -47 to -56 ), one AP- 4 (position -16 to -21 ) and two LSF (position +2 to -3 and position +12 to +8 ) binding sites are located within or near the HIV-1 LTR start site and these transcription factors recruit HDAC1 to the HIV-1 LTR $[10,11,14]$. To examine the role of these cis-acting elements and the respective relevant transcription factors in butyric acid-induced HIV-1 gene expression, we created a series of plasmid constructs of HIV-1 LTR with mutations to the three Sp1 binding sites, AP-4 
Fig. 2. The Sp1 binding site is essential for butyric acid-induced transactivation of HIV-1 LTR. (A) A schematic diagram of the HIV-1 LTR plasmid constructs with mutations to the Sp1, AP- 4 and LSF binding sites. (B) The Sp1 binding site is required for butyric acid-induced HIV-1 LTR gene expression. The HIV-1 LTR promoter constructs described in (A) were transiently transfected in HeLa cells, which were then treated with butyric acid (1.5 $\mathrm{mM}$ ) for additional $24 \mathrm{~h}$. The cells were then harvested and luciferase activity was measured. The data are presented as means \pm S.D. of triplicate experiments.

Fig. 3. Sp1 is involved in latent HIV-1 LTR gene expression. (A) Confirmation of siRNA knockdown of Sp1. TZMbl cells were transfected with $100 \mathrm{nM}$ siRNA directed against either Sp1 or GFP (control) mRNAs. After $48 \mathrm{~h}$ of transfection, cells were lysed and $\mathrm{Sp} 1$ levels were assessed by western blotting using specific antibodies. The blot was stripped and re-probed with anti$\beta$-actin antibody. (B) Inhibition of HIV1 gene expression by $\mathrm{Sp} 1$ depletion. TZM-bl cells were transfected with either Sp1 siRNA or a control. After 48 $\mathrm{h}$ of transfection, cells were either left untreated or treated with $1.5 \mathrm{mM}$ of butyric acid and incubated for additional $8 \mathrm{~h}$. Then, the cells were harvested and luciferase activity was measured. (C) Mithramycin A inhibits butyric acidstimulated HIV-1 replication from latently infected cells. ACH- 2 cells were pre-treated with mithramycin A at the indicated concentrations. After $1 \mathrm{~h}$ of
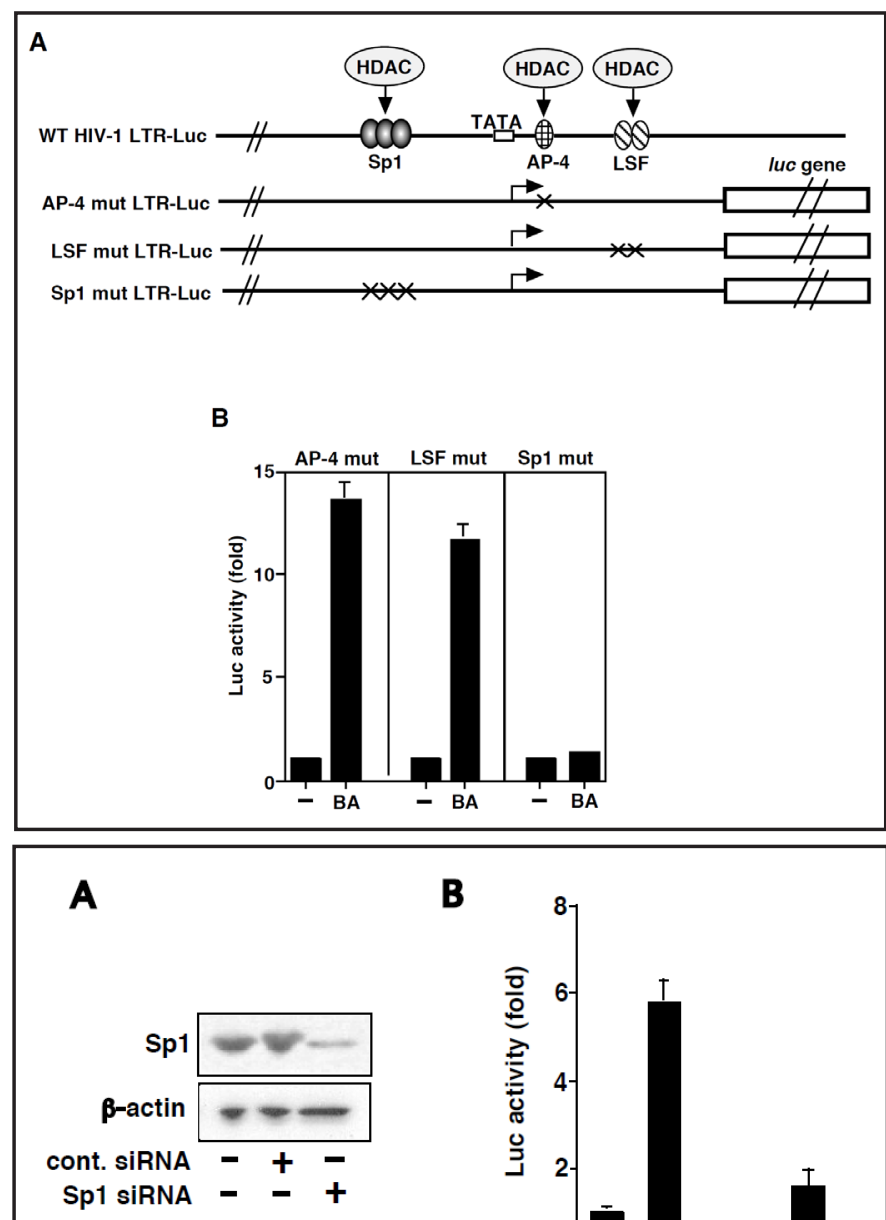

B

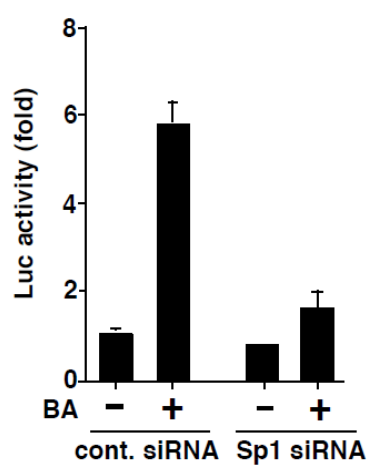

C

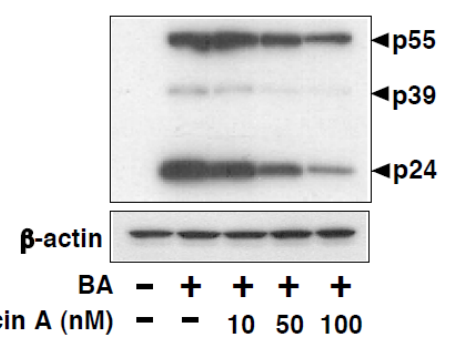

pre-treatment, cells were stimulated with butyric acid (1.5 mM) and incubated for additional $24 \mathrm{~h}$. The culture lysate was analysed for detection of HIV-1 proteins by western blotting with sera collected from AIDS patients.

binding sites and LSF binding site as described (Fig. 2A). In cells transfected with mutant LTR reporters lacking either AP-4 or LSF binding site, the extent of transcriptional induction by butyric acid was largely unchanged compared with WT LTR (Fig. 2B; compare with Fig. 1B). In contrast, transcriptional activation by butyric acid was abolished when the tandem Sp1 binding sites were mutated. These findings indicate that Sp1 appears to be the crucial cis-regulatory element with LTR for the transcriptional activation of HIV-1 by butyric acid. 
Fig. 4. CBP/p300 involvement of butyric acid-induced HIV-1 gene expression. (A) Effects of butyric acid on Sp1 promoter activation. A Sp1-luc reporter construct (20 ng) was transfected into HeLa cells, incubated for $24 \mathrm{~h}$ and stimulated with butyric acid (1, 2.5 and $5 \mathrm{mM}$ ) or TNF- $\alpha$ (1, 2 and $3 \mathrm{ng} / \mathrm{ml}$ ) for additional $24 \mathrm{~h}$. The luciferase activity of each cell lysate was then measured. (B) Effects of CBP on butyric acidinduced HIV-1 LTR gene expression. The CBP or mutant CBP (CBPAHAT) expression plasmid was co-transfected with a HIVLTR luc reporter construct into HeLa cells. After 24 h of transfection, the cells were stimulated with butyric acid (1.5 mM) for additional $24 \mathrm{~h}$, and then the luciferase activity of each cell lysate was measured. (C) CBP $\triangle$ HAT inhibits butyric acidinduced Sp1 promoter activation. The CBP $\triangle \mathrm{HAT}$ expression plasmid was co-transfected with a Sp1-luc reporter construct into HeLa cells, which were incubat-

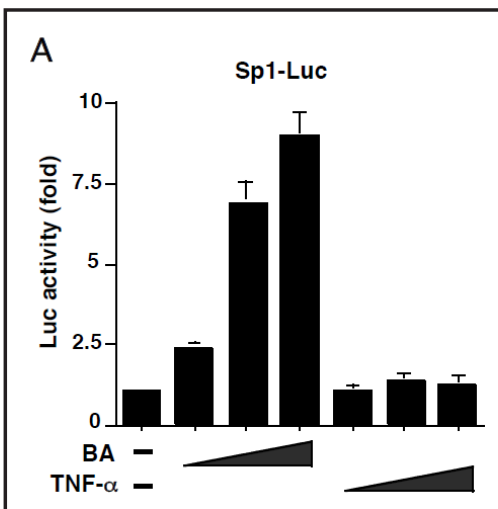

B

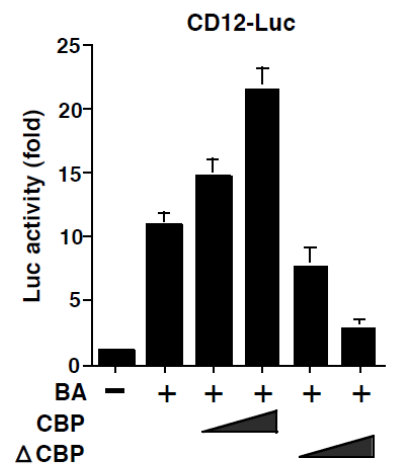

C

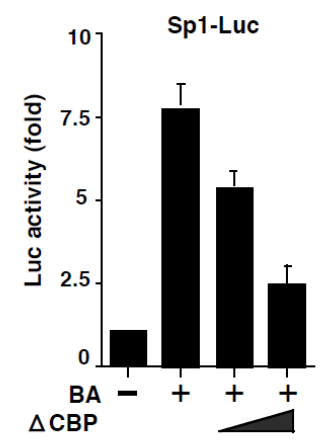

ed for $24 \mathrm{~h}$ and stimulated with butyric acid (1.5 $\mathrm{mM}$ ) for additional $24 \mathrm{~h}$. The luciferase activity of each cell lysate was then measured. (D) Inhibition of HIV-1 re-activation by C646. ACH2 cells were pre-treated with C646 (5 and $30 \mu \mathrm{M})$ for $1 \mathrm{~h}$ and then stimulated with butyric acid (1.5 mM) for additional $24 \mathrm{~h}$. The cell culture supernatants were analysed for p24 antigen levels by ELISA (upper panel). Experiments were performed in triplicate and data are presented as means and SDs ( $\mathrm{pg} / \mathrm{mL})$. Detection of various viral proteins in the cell lysate was performed by western blotting (lower panel).

The effect of Sp1 inhibition on HIV-1 gene expression

To examine the effect of endogenous Sp1, we used siRNA technique to knockdown Sp1 expression and examined HIV-1 LTR transcriptional levels after depletion of endogenous Sp1. We employed TZM-bl cells, containing a stable chromosomally integrated HIV-1 LTR $[24,25]$, to confirm the effect of butyric acid on HIV-1 transcription. We found that significant depletion of Sp1 by siRNA knockdown (Fig. 3A) resulted in downregulation of the butyric acid-induced transcription from HIV-1 LTR (Fig. 3B) without significant reduction of the cell viability (data not shown). To further confirm the involvement of Sp1 in butyric acidmediated induction of HIV-1 transcription, we used mithramycin A, a competitive antagonist for the DNA-binding of Sp1 [30], on HIV-1 replication from latently HIV-1 infected cells. In this experiment, we used the human ACH-2 cell line [22, 23], known to harbour latent HIV-1 proviruses and it is derived from human CD4 T cells and the most studied model of postintegration latency. We observed that butyric acid-induced HIV-1 replication in ACH-2 cells was greatly inhibited by mithramycin A (Fig. 3C). The similar result was observed in OM10.1 cells, a macrophage cell line chronically and latently infected with HIV-1 (data not shown). 
Fig. 5. ChIP detection of Sp1 and CBP on HIV1 LTR. (A) Dynamic association/dissociation of Sp1, HDAC1, c-myc, acetylated histone H3 (Ac-H3), RNA polymerase II (RNAPII) and CBP. TZM-bl cells were left untreated or treated with $1.5 \mathrm{mM}$ butyric acid for $90 \mathrm{~min}$, and the ChIP assay was performed using the designated antibodies. The recovered DNA was PCRamplified using HIV-1 LTR-specific primers and separated on a $2 \%$ agarose gel. Input DNA (input) represents $10 \%$ of total input chromatin DNA, and immunoprecipitation with IgG serves as a negative control. (B) Sp1 recruits CBP to the HIV-1 LTR. TZM-bl cells were transfected with siRNA targeting Sp1 or GFP, incu-
A

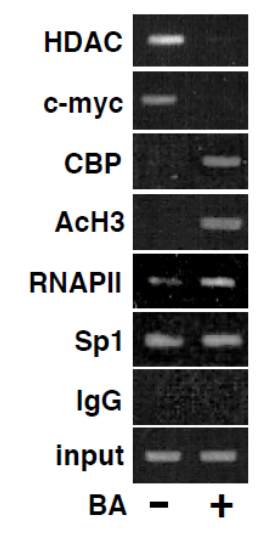

B

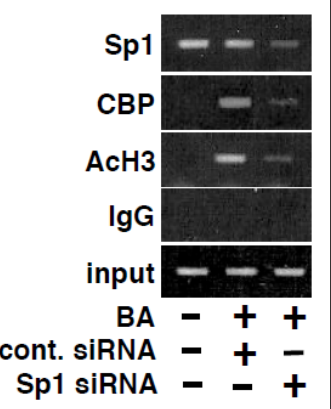

bated for $48 \mathrm{~h}$, and the association of Sp1 and CBP were analysed using the ChIP assay. The experiments were repeated with reproducible results. Representative results are shown.

These observations suggested the crucial role of Sp1 for the butyric acid-induced HIV-1 replication.

$C B P / p 300$ involvement in butyric acid-induced HIV-1 gene expression

To examine whether the effect of butyric acid on HIV-1 gene expression is solely dependent on Sp1, we utilized a synthetic promoter that is driven by three Sp1 consensus sites only (pSp1-luc) devoid of other cis-regulatory elements on HIV-1 LTR [29]. As shown in Fig. 4, when cells were transfected with pSp1-luc, the Sp1-dependent transcription activity was augmented by butyric acid in a dose-dependent manner. However, this effect was not observed with TNF- $\alpha$ (Fig. 4) confirming the result of Fig. 1. Previous studies have shown that $\mathrm{Sp} 1$ sites can recruit transcriptional co-activators such as cAMP response elementbinding (CREB)-binding protein (CBP)/p300 as well as repressor factors/complexes [31, 32]. In addition, butyric acid treatment promoted the histone acetylation in latently HIV-1 infected cells $[20,21]$. Thus it was suggested that derepression of HDAC activity by butyric acid appears to convert the action of Sp1 from transcriptional repressor to activator through subsequent recruitment of transcriptional co-activators such as CBP/p300 instead of HDAC1.

We then examined the effect of CBP overexpression on the butyric acid-induced HIV-1 gene expression. As shown in Fig. 4B, CBP overexpression dose-dependently augmented the butyric acid-induced HIV-1 LTR gene expression. In contrast, overexpression of a mutant CBP lacking histone acetyltransferase (HAT) activity (CBPAHAT) inhibited the butyric acidinduced HIV-1 gene expression (Fig. 4B). CBP $\triangle$ HAT also inhibited butyric acid-induced pSp1luc activity (Fig. 4C). To further investigate the involvement of CBP in butyric acid-mediated activation, we used C646 a specific inhibitor of CBP/p300 HAT [33]. As demonstrated in Fig. $4 \mathrm{D}$, the butyric acid-induced viral production was dramatically downregulated either in the culture supernatant (upper panel) or within the cytoplasm (lower panel) upon treatment of ACH-2 cells with $\mathrm{C} 646$. These results suggest that CBP is involved in the butyric acid-induces HIV-1 transcription at least in a part.

\section{ChIP analysis to detect Sp1 and CBP on HIV-1 LTR}

The above results suggest that $\mathrm{Sp} 1$ is solely responsible for the butyric acid-mediated transcriptional induction from HIV-1 LTR through recruitment of transcriptional coactivators such as CBP. To further confirm the role of Sp1 in the recruitment of CBP, we performed ChIP assay using specific antibodies with TZM-bl cells. When TZM-bl cells were treated with butyric acid to induce HIV-1 transcription, we observed dissociation of HDAC1 and c-Myc from the HIV-1 promoter (Fig. 5A). In contrast, CBP was recruited to the HIV-1 LTR upon 
butyric acid treatment concomitantly with the recruitment of Ac-H3 and RNAPII to the viral promoter. Interestingly, the amount of Sp1 recruited to the HIV LTR remained unchanged by butyric acid treatment. To further confirm whether Sp1 is essential for the recruitment of CBP to the HIV-1 promoter, we examined the effect of Sp1 knockdown on the HIV-1 LTR transcription (Fig. 5B). Depletion of Sp1 by siRNA resulted in the abrogation of CBP recruitment and the local H3 acetylation in the vicinity of HIV-1 promoter. These results have indicated that inhibition of Sp1-associated HDAC activity by butyric acid is essential for the CBP recruitment to the HIV-1 promoter and induction of viral transcription.

\section{Discussion}

The ability of HIV-1 to establish the latent infection and its occasional re-activation is considered crucial for the progression of HIV-1-associated diseases. Thus, elucidation of the molecular mechanism that regulates the viral gene expression is essential for understanding the HIV-1 latency and for developing novel therapy against HIV-1 infection to prevent disease progression to AIDS. It is now clear that HDACIs could stimulate replication from HIV-1 provirus through transcriptional induction from the proviral LTR associated with histone deacetylation $[2,6,9]$. It was demonstrated that metabolites produced from anaerobic bacteria such as Porphyromonas gingivalis, Fusobacterium nucleatum and Clostridium cochlearium are responsible for this HDACI activity [20, 21, 34]. Among the short chain (C2-C5) fatty acids (SCFAs) metabolites produced from anaerobic bacteria, butyric acid demonstrated the most potent activity as an HDACI and exhibited a number of distinctive biological activities when added to live cells $[15,16,18]$. We reported previously that butyric acid is the major SCFA for stimulation of HIV-1 gene expression in the latently infected cells and suggested that microbiological control of infection by such bacteria upon periodontitis or change of gut flora should prevent the HIV-1 disease progression [20,21, 34]. Here we have provided scientific evidence to further clarify the molecular mechanism by which butyric acid activates latently infected HIV-1.

There are a number of distinct cis-regulatory elements within the HIV-1 LTR to which respective trans-acting factors interact and co-ordinately regulate viral transcription. Among the host transcription factors that are known to recruit HDAC proteins to the HIV-1 LTR thus being involved in the maintenance of HIV-1 latency [10-14], we found that the Sp1 sites among other cis-regulatory elements were responsible for the butyric acid-induced gene expression (Fig. 1 and 2). This finding was confirmed by the experiment using Sp1-specific promoter (Fig. 4) and Sp1 depletion using the specific siRNA (Fig. 3). In addition, a specific Sp1 inhibitor compound mithramycin A blocked the butyric acid-mediated induction of HIV1 viral replication from the latently infected cells (Fig. 3). Thus we concluded that Sp1 is primarily responsible for the effect of butyric acid.

Sp1 binding sites, rich in GC sequences, are ubiquitously present in many cellular and viral promoters. In fact, Sp1 binding sites are occupied during the transcription of large number of genes and actively involved in their gene expression [35-37]. Although it is still too premature to conclude that the presence of Sp1 binding sites is prerequisite for the responsiveness to butyric acid, a previous study reported poor butyrate activation of some cellulargenes including the p21/WAF1/Cip1 and death receptor 5 genes that lack Sp1 binding sites in the promoter $[38,39]$. Choudhary et al. reported that hexamethylbisacetamide could activate HIV-1 expression depending on the presence of the Sp1 site through recruitment of CDK9, a cartalytic subunit of P-TEFb that is essential for the Tat-mediated trans-activation of HIV-1, in the absence of NF- $\mathrm{kB}$ binding site [40]. Thus, together with our findings, the butyric acid-induced HIV-1 gene expression is attributable to Sp1. Furthermore, it was reported that butyrate-induced HIV-1 LTR expression does not require de novo protein synthesis [17]. Interestingly, $\mathrm{Sp} 1$ was required for the transcriptional repression mediated by c-Myc acting as a docking molecule between c-Myc and HDAC1 [11]. In our experiment with ChIP assay, butyric acid administration disrupted the formation of repressor complex by displacing 
HDAC1 from the HIV-1 promoter region (Fig. 5). Thus, Sp1 might act as a docking molecule between transcription factors and transcriptional co-regulators either co-activators or corepressors.

We further found that the presence of transcriptional coactivator CBP/p300 and its HAT activity was indispensable by the experiments using CBP mutant lacking HAT activity (CBP $\triangle H A T$ ) and a specific inhibitor for CBP/p300 (Fig. 4 and 5). Upon the treatment with butyric acid, the enzymatically active CBP was recruited to the HIV-1 LTR as evidenced by the appearance of the acetylated form of H3 in the HIV-1 promoter (Fig. 5). Furthermore, siRNA-mediated Sp1 depletion resulted in the decreased recruitment of CBP to the promoter (Fig. 5). These observations suggested that CBP competes with HDAC1 binding to Sp1 upon treatment with butyric acid, thus abolishing HDAC1-mediated repression of HIV-1 LTR.

It is well established that HIV-1 gene regulation depends on reversible acetylation of histones and subsequent changes in the chromatin structure. Previous studies demonstrated that Sp1 could act as both positive and negative regulator of gene expression in the context of transcriptional regulatory circuit, wherein Sp1 can directly interact with either HATs, a transcriptional coactivator, or a transcriptional repressor, such as HDAC [31, 32, 36]. In addition, several nuclear HATs, such as CBP/p300 and p300/CBP-associated factor, are positively involved in HIV-1 transcription [6, 9]. Moreover, the minimum LTR promoter (CD52 Luc) and even the Sp1 Luc reporter plasmid were susceptible to the butyric acid-induced transcriptional activation (Fig. 1 and 4). Thus, it is suggested that butyric acid could activate Sp1-dependent transcription by altering chromatin modification via HATs through inhibiting HDAC activity. In addition, previous reports indicated that acetylation of Sp1 influences its transcriptional activity and protein stability [36, 41]. Moreover, Sp1 was shown to contribute to the recruitment of a positive transcription elongation factor complex to the HIV-1 promoter [40]. It is possible that such biochemical modification of general transcription factors or mediators might determine the direction of transcriptional regulation and play a key role in this drastic transcriptional conversion. Thus, Sp1 represents a modular molecule that specifically target HDACs and HATs at the HIV-1 promoter, regulating histone acetylation status. Further studies are warranted to elucidate the molecular mechanism and the direct target molecule of butyric acid-mediated de-acetylation that converts transcriptionallysilent Sp1 to its active form.

The biological and clinical implication of this butyric acid-mediated transcriptional control of HIV-1 replication might explain why periodontitis is strongly correlated with the progression of HIV-1 infection [34, 42, 43]. There are a number of butyric acid-producing bacteria in the gut and vagina, both of which are major sites of HIV-1 transmission and its replication [44-49]. In fact, it is known that infection of butyric acid-producing bacteria is involved in AIDS progression and causally related to the re-activation of the latent HIV-1 provirus $[20,21,47,50,51]$. Further elucidation of the molecular mechanism by which butyric acid activates the latent HIV-1 provirus and basic studies of butyrate-producing bacteria should facilitate the development of new measures to prevent the clinical progression of HIV-1 infection though developing specific small molecular compounds or even probiotics approaches.

\section{Acknowledgements}

This work was supported by Grants-in-aid from the Ministry of Health, Labour and Welfare of Japan, the Ministry of Education, Culture, Sports, Science and Technology (MEXT) of Japan, the Dental Research Center, Nihon University School of Dentistry (Tokyo), the IMAI MEMORIAL TRUST FOR AIDS RESEARCH and the Strategic Research Base Development Program for Private Universities from the MEXT of Japan, 2010-2014 (grant no.: S1001024). 


\section{Disclosure Statement}

The authors confirm that there are no conflicts of interest.

\section{References}

1 Coiras M, Lopez-Huertas MR, Perez-Olmeda M, Alcami J: Understanding HIV-1 latency provides clues for the eradication of long-term reservoirs. Nat Rev Microbiol 2009;7:798-812.

2 Marcello A: Latency: The hidden HIV-1 challenge. Retrovirology 2006;3:7.

3 Siliciano RF: Scientific rationale for antiretroviral therapy in 2005: Viral reservoirs and resistance evolution. Top HIV med 2005;13:96-100.

4 Siliciano JD, Kajdas J, Finzi D, Quinn TC, Chadwick K, Margolick JB, Kovacs C, Gange SJ, Siliciano RF: Longterm follow-up studies confirm the stability of the latent reservoir for HIV-1 in resting CD4+ T cells. Nat med 2003;9:727-728.

5 Bisgrove D, Lewinski M, Bushman F, Verdin E: Molecular mechanisms of HIV-1 proviral latency. Expert Rev Anti infect Ther 2005;3:805-814.

6 Colin L, Van Lint C: Molecular control of HIV-1 postintegration latency: Implications for the development of new therapeutic strategies. Retrovirology 2009;6:111.

7 Okamoto T, Wong-Staal F: Demonstration of virus-specific transcriptional activator(s) in cells infected with HTLV-III by an in vitro cell-free system. Cell 1986;47:29-35.

8 Van Lint C, Bouchat S, Marcello A: HIV-1 transcription and latency: An update. Retrovirology 2013;10:67.

9 Mbonye U, Karn J: Transcriptional control of hiv latency: Cellular signaling pathways, epigenetics, happenstance and the hope for a cure. Virology 2014;454-455:328-339.

10 Coull JJ, Romerio F, Sun JM, Volker JL, Galvin KM, Davie JR, Shi Y, Hansen U, Margolis DM: The human factors YY1 and LSF repress the human immunodeficiency virus type 1 long terminal repeat via recruitment of histone deacetylase 1. J Virol 2000;74:6790-6799.

11 Jiang G, Espeseth A, Hazuda DJ, Margolis DM: C-myc and Sp1 contribute to proviral latency by recruiting histone deacetylase 1 to the human immunodeficiency virus type 1 promoter. J Virol 2007;81:1091410923.

12 Williams SA, Chen LF, Kwon H, Ruiz-Jarabo CM, Verdin E, Greene WC: NF-kappaB p50 promotes HIV latency through hdac recruitment and repression of transcriptional initiation. EMBO J 2006;25:139-149.

13 Tyagi M, Karn J: CBF-1 promotes transcriptional silencing during the establishment of HIV-1 latency. EMBO J 2007;26:4985-4995.

14 Imai K, Okamoto T: Transcriptional repression of human immunodeficiency virus type 1 by AP-4. J Biol Chem 2006;281:12495-12505.

15 Louis P, Flint HJ: Diversity, metabolism and microbial ecology of butyrate-producing bacteria from the human large intestine. FEMS Microbiol Lett 2009;294:1-8.

16 Berni Canani R, Di Costanzo M, Leone L: The epigenetic effects of butyrate: Potential therapeutic implications for clinical practice. Clin epigenetics 2012;4:4.

17 Laughlin MA, Zeichner S, Kolson D, Alwine JC, Seshamma T, Pomerantz RJ, Gonzalez-Scarano F: Sodium butyrate treatment of cells latently infected with HIV-1 results in the expression of unspliced viral RNA. Virology 1993;196:496-505.

18 Riggs MG, Whittaker RG, Neumann JR, Ingram VM: N-butyrate causes histone modification in HeLa and friend erythroleukaemia cells. Nature 1977;268:462-464.

19 Kashanchi F, Melpolder JC, Epstein JS, Sadaie MR: Rapid and sensitive detection of cell-associated HIV-1 in latently infected cell lines and in patient cells using sodium-n-butyrate induction and RT-PCR. J Med Virol 1997;52:179-189.

20 Imai K, Ochiai K, Okamoto T: Reactivation of latent HIV-1 infection by the periodontopathic bacterium Porphyromonas gingivalis involves histone modification. J Immunol 2009;182:3688-3695.

21 Imai K, Yamada K, Tamura M, Ochiai K, Okamoto T: Reactivation of latent HIV-1 by a wide variety of butyric acid-producing bacteria. Cellular and molecular life sciences : Cell Mol Life Sci 2012;69:2583-2592. 
22 Clouse KA, Powell D, Washington I, Poli G, Strebel K, Farrar W, Barstad P, Kovacs J, Fauci AS, Folks TM: Monokine regulation of human immunodeficiency virus-1 expression in a chronically infected human $\mathrm{T}$ cell clone. J Immunol 1989;142:431-438.

23 Folks TM, Clouse KA, Justement J, Rabson A, Duh E, Kehrl JH, Fauci AS: Tumor necrosis factor $\alpha$ induces expression of human immunodeficiency virus in a chronically infected T-cell clone. Proc Natl Acad Sci USA 1989;86:2365-2368.

24 Derdeyn CA, Decker JM, Sfakianos JN, Wu X, O'Brien WA, Ratner L, Kappes JC, Shaw GM, Hunter E: Sensitivity of human immunodeficiency virus type 1 to the fusion inhibitor T-20 is modulated by coreceptor specificity defined by the V3 loop of gp120. J Virol 2000;74:8358-8367.

25 Platt EJ, Wehrly K, Kuhmann SE, Chesebro B, Kabat D: Effects of CCR5 and CD4 cell surface concentrations on infections by macrophagetropic isolates of human immunodeficiency virus type 1. J Virol 1998;72:28552864.

26 Imai K, Togami H, Okamoto T: Involvement of histone H3 lysine 9 (H3K9) methyltransferase G9a in the maintenance of hiv-1 latency and its reactivation by bix01294. J Biol Chem 2010;285:16538-16545.

27 Takada N, Sanda T, Okamoto H, Yang JP, Asamitsu K, Sarol L, Kimura G, Uranishi H, Tetsuka T, Okamoto T: RelA-associated inhibitor blocks transcription of human immunodeficiency virus type 1 by inhibiting NFkappaB and sp1 actions. J Virol 2002;76:8019-8030.

28 Victoriano AF, Imai K, Togami H, Ueno T, Asamitsu K, Suzuki T, Miyata N, Ochiai K, Okamoto T: Novel histone deacetylase inhibitor NCH-51 activates latent HIV-1 gene expression. FEBS Lett 2011;585:1103-1111.

29 Torigoe T, Izumi H, Yoshida Y, Ishiguchi H, Okamoto T, Itoh H, Kohno K: Low PH enhances Sp1 DNA binding activity and interaction with TBP. Nucleic Acids Res 2003;31:4523-4530.

30 Blume SW, Snyder RC, Ray R, Thomas S, Koller CA, Miller DM: Mithramycin inhibits Sp1 binding and selectively inhibits transcriptional activity of the dihydrofolate reductase gene in vitro and in vivo. J Clin Invest 1991;88:1613-1621.

31 Huang W, Zhao S, Ammanamanchi S, Brattain M, Venkatasubbarao K, Freeman JW: Trichostatin a induces transforming growth factor beta type II receptor promoter activity and acetylation of Sp1 by recruitment of PCAF/p300 to a Sp1/NF-Y complex. J Biol Chem 2005;280:10047-10054.

32 Jang SI, Steinert PM: Loricrin expression in cultured human keratinocytes is controlled by a complex interplay between transcription factors of the Sp1, CREB, AP1, and AP2 families. J Biol Chem 2002;277:42268-42279.

33 Bowers EM, Yan G, Mukherjee C, Orry A, Wang L, Holbert MA, Crump NT, Hazzalin CA, Liszczak G, Yuan H, Larocca C, Saldanha SA, Abagyan R, Sun Y, Meyers DJ, Marmorstein R, Mahadevan LC, Alani RM, Cole PA: Virtual ligand screening of the p300/CBP histone acetyltransferase: Identification of a selective small molecule inhibitor. Chem Biol 2010;17:471-482.

34 Imai K, Victoriano AF, Ochiai K, Okamoto T: Microbial interaction of periodontopathic bacterium Porphyromonas gingivalis and HIV-possible causal link of periodontal diseases to AIDS progression. Curr HIV Res 2012;10:238-244.

35 Liu Z, Simpson ER: Molecular mechanism for cooperation between Sp1 and steroidogenic factor-1 (SF-1) to regulate bovine CYP11A gene expression. Mol Cell Endocrinol 1999;153:183-196.

36 Tan NY, Khachigian LM: Sp1 phosphorylation and its regulation of gene transcription. Mol Cell Biol 2009;29:2483-2488.

37 Burnett JC, Miller-Jensen K, Shah PS, Arkin AP, Schaffer DV: Control of stochastic gene expression by host factors at the HIV promoter. PLoS Pathog 2009;5:e1000260.

38 Nakano K, Mizuno T, Sowa Y, Orita T, Yoshino T, Okuyama Y, Fujita T, Ohtani-Fujita N, Matsukawa Y, Tokino T, Yamagishi H, Oka T, Nomura H, Sakai T: Butyrate activates the WAF1/Cip1 gene promoter through Sp1 sites in a p53-negative human colon cancer cell line. J Biol Chem 1997;272:22199-22206.

39 Yoshida T, Maeda A, Tani N, Sakai T: Promoter structure and transcription initiation sites of the human death receptor 5/ TRAIL-R2 gene. FEBS lett 2001;507:381-385.

40 Choudhary SK, Archin NM, Margolis DM: Hexamethylbisacetamide and disruption of human immunodeficiency virus type 1 latency in CD4(+) t cells. J Infect Dis 2008;197:1162-1170.

41 Chu S: Transcriptional regulation by post-transcriptional modification -role of phosphorylation in sp1 transcriptional activity. Gene 2012;508:1-8. 


\section{Cellular Physiology Cell Physiol Biochem 2015;37:853-865

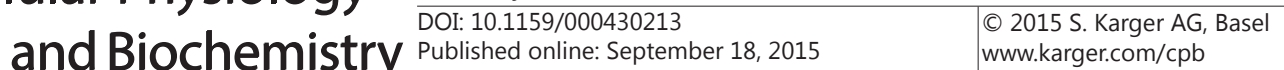 \\ Imai et al.: Sp1 Plays a Key Role in Butyric Acid-Mediated HIV-1 Activation}

42 Maticic M, Poljak M, Kramar B, Tomazic J, Vidmar L, Zakotnik B, Skaleric U: Proviral HIV-1 DNA in gingival crevicular fluid of HIV-1-infected patients in various stages of HIV disease. J Dent Res 2000;79:1496-1501.

43 Shugars DC, Slade GD, Patton LL, Fiscus SA: Oral and systemic factors associated with increased levels of human immunodeficiency virus type 1 RNA in saliva. Oral Surg, Oral Med, Oral Pathol, Oral Radiol Endod 2000;89:432-440.

44 Cummings JH, Pomare EW, Branch WJ, Naylor CP, Macfarlane GT: Short chain fatty acids in human large intestine, portal, hepatic and venous blood. Gut 1987;28:1221-1227.

45 Mortensen PB, Clausen MR: Short-chain fatty acids in the human colon: Relation to gastrointestinal health and disease. Scand J Gastroenterol Suppl 1996;216:132-148.

46 Pryde SE, Duncan SH, Hold GL, Stewart CS, Flint HJ: The microbiology of butyrate formation in the human colon. FEMS Microbiol Lett 2002;217:133-139.

47 Kantor B, Ma H, Webster-Cyriaque J, Monahan PE, Kafri T: Epigenetic activation of unintegrated HIV-1 genomes by gut-associated short chain fatty acids and its implications for HIV infection. Proc Natl Acad Sci USA 2009;106:18786-18791.

48 Murdoch DA: Gram-positive anaerobic cocci. Clin Microbiol Rev 1998;11:81-120.

49 Stein TP, Koerner B, Schluter MD, Leskiw MJ, Gaprindachvilli T, Richards EW, Cope FO, Condolucci D: Weight loss, the gut and the inflammatory response in aids patients. Cytokine 1997;9:143-147.

50 Gray RH, Wawer MJ, Sewankambo N, Serwadda D: HIV-1 infection associated with abnormal vaginal flora morphology and bacterial vaginosis. Lancet 1997;350:1780.

51 Taha TE, Gray RH, Kumwenda NI, Hoover DR, Mtimavalye LA, Liomba GN, Chiphangwi JD, Dallabetta GA, Miotti PG: HIV infection and disturbances of vaginal flora during pregnancy. J Acquir Immune Defic Syndr Hum Retrovirol 1999;20:52-59. 